\title{
3. Darstellung der Aldehyde aus den Säuren $\mathrm{C}_{\mathrm{n}} \mathrm{H}_{\mathrm{n}} \mathrm{O}_{4}$.
}

Man weifs schon lange, dafs beim Erhitzen vieler Salze der Säuren $\mathrm{C}_{\mathrm{n}} \mathrm{H}_{\mathrm{n}} \mathrm{O}_{4}$ gleichzeitig mit dem Aceton eine den Aldehyden isomerische und in vielen Eigenschaften ähnliche Verbindung auftritt (Propylal, Butyral und Valeral); aber Aldehyde, wie sie z. B. aus den Alkoholen durch Oxydation gewonnen werden, hat man bis jetzt noch nicht aus den Säuren dargestellt.

Aus den heute gültigen Ansichten über die Constitution der Säuren $\mathrm{C}_{n} \mathrm{H}_{n} \mathrm{O}_{4}$, der Acetone und Aldehyde, so wie aus der Zersetzung, welche bei Bildung der Acetone stattindet, kann man folgern, dafs durch einen analogen Procels die Aldehyde entstehen müssen.

Zur Bildung der Acetone sind immer 2 Aeq. des Salzes erforderlich :

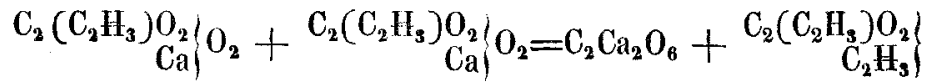

Essigs. Kalk.

Essigs. Kalk.

Aceton.

Von dem einen Aequivalent des essigsauren Kalks bleibt nur das Radical, Acetyl oder Formyl-Methyl, von dem zweiten nur das Methyl, welche sich zu dem Aceton vereinigen, während die übrigen Elemente kohlensauren Kalk bilden.

Erhitzt man ein Gemisch der Salze zweier verschiedener Säuren, z. B. valeriansauren Kalk mit essigsaurem Kalk, so tritt dieselbe Metamorphose ein; es entsteht 1 Aeq. des kohlensauren Kalks und ein Aceton, der aus dem Radical der einen Säure und dem im Radical der andern Säure befindlichen Alkoholradical zusammengesetzt ist :

$\left.\left.\mathrm{C}_{2}\left(\mathrm{C}_{2} \mathrm{H}_{3}\right) \underset{\mathrm{Ca}}{\mathrm{O}_{2}}\right\} \mathrm{O}_{2}+{ }^{\mathrm{C}_{3}\left(\mathrm{C}_{8} \mathrm{H}_{9}\right) \mathrm{Ca}_{2}}\right\} \mathrm{O}_{2}=\mathrm{C}_{2} \mathrm{Ca}_{2} \mathrm{O}_{6}+{ }^{\mathrm{C}_{2}\left(\mathrm{C}_{2} \mathrm{H}_{3}\right) \mathrm{C}_{8} \mathrm{CH}_{9}}$ oder $\mathrm{C}_{3}\left(\mathrm{C}_{3} \mathrm{H}_{9}\right) \mathrm{O}_{2}$ Essig. Kalk. Valerians, Kalk.

Mischt man nun ameisensauren Kalk mit den Kalksalzen anderer Säuren der Reihe $\mathrm{C}_{n} \boldsymbol{H}_{n} \mathrm{O}_{4}$ und unterwirft das Gemenge 
der Destillation, so entsteht ein Aceton, welcher an der Stelle des Alkoholradicals ein Aequivalent Wasserstoff enthält, d. h. ein Aldehyd :

$$
\left.\mathrm{C}_{2}\left(\mathrm{C}_{2} \mathrm{H}_{3}\right) \underset{\mathrm{Ca}}{\mathrm{O}_{2}} \mathrm{O}_{2}+\underset{\mathrm{Ca}}{\mathrm{C}_{2}(\mathrm{H}) \mathrm{O}_{2}} \mathrm{O}_{2}=\mathrm{C}_{2} \mathrm{Ca}_{2} \mathrm{O}_{6}+{ }_{2}^{\mathrm{C}_{2}\left(\mathrm{C}_{2} \mathrm{H}_{3}\right) \mathrm{O}_{2}} \mathrm{H}\right\}
$$

$$
\text { Essigs. Kalk. Ameisens. Kalk. Acetylaldehyd. }
$$

Herr Ritter hat mehrere Aldehyde nach dieser Methode dargestellt und ich lasse hier die Resultale seiner Untersuchung folgen.

Acetylaldehyd $\mathrm{C}_{4} \mathrm{H}_{4} \mathrm{O}_{2}$. - Trockener ameisensaurer Kalk und essigsaurer Kalk, in äquivalenten Mengen gemischt, wurden in einer Retorte über freiem Feuer erhitzt; das Destillat wurde in kalt gehaltenem Aether aufgefangen und mit trockenem Ammoniakgas gesältigt, worauf sich Krystalle von Aldehyd-Ammoniak in reichlicher Menge ausschieden. - Es traten bei der Bereitung des Acetylaldehyds, namentlich wenn die Operation mit gröfseren Mengen ausgeführt wurde, brenzliche Producte auf, deren Entstehung theils der unvollkommenen Mischung, theils der zu starken Erhitzung zuzuschreiben sind.

Propylaldehyd $\mathrm{C}_{6} \mathrm{H}_{8} \mathrm{O}_{2}$. - Aus Cyanäthyl dargestellter propionsaurer Baryt wurde mit ameisensaurem Kalk gemengt und destillirt. Es konnten nur geringe Mengen zum Versuch angewandt werden und das wenige Grammen betragende Destillat mufste defshalb nach zweimaliger Rectification, bei welcher jedesmal das zuerst Uebergehende gesammelt wurde, der Analyse unterworfen werden. Die Abscheidung des Propylaldehyds mit saurem schwefligsaurem Alkali konnte hier nicht versucht werden, da eine Verunreinigung mit Propionon wahrscheinlich war. Der Siedepunkt des zur Analyse benutzten Propylaldehyds lag zwischen 48 und $55^{\circ}$ (das aus dem butleressigsauren Kalk erhaltene Propylal siedet bei $\left.66^{\circ}\right)$. 
I. 0,1403 Grm. Substanz lieferten 0,2985 Grm. Kohlensäure und 0,1295 Grm. Wasser.

II. 0,1739 Grm. Substanz lieferten 0,3702 Grm. Kohlensäure und 0,1595 Grm. Wasser.

\begin{tabular}{crrcc} 
& \multicolumn{2}{c}{} & \multicolumn{2}{c}{ gefunden } \\
& \multicolumn{2}{c}{ berechnet } & I. & II. \\
$\mathrm{C}_{6}$ & 36 & 62,0 & 58,02 & 58,05 \\
$\mathrm{H}_{6}$ & 6 & 10,3 & 10,33 & 10,29 \\
$\mathrm{O}_{2}$ & 16 & 27,7 & - & -
\end{tabular}

So wenig der gefundene Kohlenstoff mit der Rechnung stimmt, so scheint die Analyse doch zu beweisen, dafs nach obiger Methode Propylaldehyd erhalten werden kann (die bei der Analyse gefundenen Zahlen ergeben das Verhältnifs der Elemente $\mathrm{C}: \mathrm{H}: 0=6: 6,2: 2,5)$.

Valeraldehyd $\mathrm{C}_{10} \mathrm{H}_{10} \mathrm{O}_{2}$. - Ameisensaurer Kalk und valeriansaurer Kalk wurden destillirt. Aus dem bei etwa $100^{\circ}$ siedenden Theil des Destillats wurde schwefligsaures Valeraldehyd-Natrium dargestellt und analysirt.

2,543 Grm. Substanz lieferten nach der Zerstörung mit Salzsäure und chlorsaurem Kali 2,936 Grm. schwefelsauren Baryt, woraus sich für das Salz 31,71 pC. schweflige Säure berechnen; die Formel $\mathrm{C}_{10} \mathrm{H}_{9} \mathrm{NaS}_{2} \mathrm{O}_{6}+3$ aq. verlangt $32,13 \mathrm{pC}$. schweflige Säure.

Oenanthol $\mathrm{C}_{14} \mathrm{H}_{14} \mathrm{O}_{2}$ - - Bei der Destillation des önanthylsauren Kalks mit ameisensaurem Kalk wurde ein Oel erhalten, das mit saurem schwefligsaurem Natron sich zu einer in Blättchen krystallisirenden Verbindung vereinigte; diese hatte das Ansehen des schwefligsauren OenanthylNatriums und das mit Säuren daraus abgeschiedene Oel besafs den Geruch des Oenanthols.

Caprylaldehyd $\mathrm{C}_{16} \mathrm{H}_{16} \mathrm{O}_{2}$. - Das aus caprylsaurem Kalk und ameisensaurem Kalk erhaltene Destillat besafs den 
Geruch des Caprylaldehyds und vereinigte sich mit saurem schwefligsaurem Natron zu einer krystallinischen Verbindung.

Myristinsaurer und palmitinsaurer Kalk lieferten bei Destillation mit ameisensaurem Kalk sehr widerlich riechende Producte, die zum gröfsten Theil ölig waren und nur geringe Mengen fester Substanz absetzten. Letztere bestand nach dem Abpressen und mehrmaligem Umkrystallisiren aus heifsem Weingeist aus vollkommen weilsen, geruchlosen kleinen Krystallschuppen, gab aber bei der Verbrennung bei weitem mehr Kohlenstoff und Wasserstoff, als dem Myristinaldehyd und Palmitinaldehyd entspricht. Es ist wahrscheinlich, dafs bei der hohen Temperatur, welche zur Zersetzung der Salzgemenge nothwendig ist, diese Aldehyde nicht bestehen können.

Die Darstellung der Aldehyde nach dieser Methode wird vielleicht nur selten vortheilhaft sein, denn es treten gleichzeitig brenzliche Producte in ziemlich bedeutender Menge auf, und diese erlaubten auch nicht, das Oenanthol und den Caprylaldehyd zur Analyse genügend zu reinigen, oder es hätte der Versuch in weit gröfserem Mafsstabe angestellt werden müssen. Vielleicht vermeidet man diese Verunreinigung durch Erhitzen des Salzgemisches in einem Oelbade, indem man die zur Zersetzung nöthige Temperatur möglichst wenig überschreitet.

\section{Ueber das Ozon; nach Th. Andrews*).}

Andrews hat Untersuchungen über das 0zon ausgeführt, namentlich mit Rücksicht darauf, ob, wie aus neueren Unter-

*) Chem. Gaz. 1855, 339. 